\title{
Fatores associados ao insucesso do tratamento da leishmaniose cutânea com antimoniato de meglumina
}

\author{
Factors associated with treatmet failure of cutaneous leishmaniasis \\ with meglumine antimoniate
}

\author{
Alex Miranda Rodrigues ${ }^{1,2}$, Márcia Hueb ${ }^{3}$, Thiago Adler Ralho Rodrigues dos Santos ${ }^{3}$ \\ e Cor Jésus Fernandes Fontes ${ }^{1,3}$
}

\begin{abstract}
RESUMO
Foram investigados os fatores associados ao insucesso do tratamento da leishmaniose cutânea com antimoniato de meglumina num serviço de referência para leishmanioses, em Mato Grosso. Uma coorte histórica de 151 pacientes com diagnóstico de leishmaniose cutânea foi construída com informações dos prontuários. A incidência de insucesso após o primeiro ciclo de antimonial foi $47 \%$ (IC95\%=39,2\%-55\%). Dose de antimonial inferior a 10mg/kg/dia $(R R=1,8 ;$ IC95:1,1-3,0), tratamento prévio para leishmaniose ( $R R=1,7$; IC95:1,3-2,4), três ou mais lesões ( $R R=1,9 ;$ IC95:1,4-2,5), tratamento irregular $(R R=1,9 ;$ IC95:1,3-2,6) e peso maior que 68kg ( $R R=1,7 ;$ IC95:1,1-2,5) foram associados ao insucesso terapêutico. Após ajuste, permaneceram associados ao insucesso os seguintes fatores: 3 ou mais lesões cutâneas $(O R=4,6 ;$ IC95\%=1,2-17,4), tratamento anterior para leishmaniose tegumentar americana $(O R=4,5 ;$ IC95\%=1,1-7,5), peso maior que $68 \mathrm{~kg}$ $(O R=4,3$; IC95\%=1,5-11,9) e irregularidade no tratamento $(O R=12,5$; IC95\%=2,1-75,4), embora o peso possivelmente tenha sido associado ao insucesso devido à limitação da dose máxima. Estes achados auxiliam na identificação de pacientes com maior risco de insucesso no tratamento da leishmaniose cutânea com antimonial.
\end{abstract}

Palavras-chaves: Leishmaniose cutânea. Antimoniato de meglumina. Prognóstico. Tratamento. Mato Grosso.

\begin{abstract}
We investigated factors associated with treatment failure in the treatment of cutaneous leishmaniasis with meglumine antimony in a reference service in Mato Grosso State. A retrospective cohort of 151 patients with cutaneous leishmaniasis was built using medical records. The incidence of therapeutic failure was 47\% (IC95\%=39.2\%-55\%). Antimoniate doses below 10mg $/ \mathrm{kg} / \mathrm{day}$ ( $R R=1.8$; IC95:1.1-3.0), previous leishmaniasis treatment ( $R R=1.7 ;$ IC95:1.3-2.4), 3 or more skin lesions (RR=1.9; IC95:1.4-2.5), incomplete treatment $(R R=1.9$; IC95:1.3-2.6) and body weight above $68 \mathrm{~kg}(R R=1.7 ;$ IC95:1.1-2.5) were associated with therapeutic failure. After adjustment, therapeutic failure was associated with having 3 or more cutaneous lesions $(O R=4.6 ; I C 95 \%=1.2-17.4)$, reports of previous leishmaniasis treatment $($ OR=4.5; IC95\%=1.1-17.5), body weight above $68 \mathrm{~kg}(\mathrm{OR}=4.3$; IC95=1.5-11.9) and incomplete treatment schedule $(O R=12.5$; IC95\%=2.1-75.4), although body weight is possibly associated with treatment failure due to the limitation of the maximum daily dose. These results help to identify patients at risk of treatment failure of cutaneous leishmaniasis with antimony therapy.
\end{abstract}

Key-words: Cutaneous leishmaniasis. Meglumine antimoniate. Prognosis. Treatment. Mato Grosso.

A leishmaniose tegumentar americana (LTA) é uma doença infecciosa, não contagiosa de pele e mucosas, causada por protozoários do gênero Leishmania ${ }^{211}$. É uma infecção zoonótica que afeta vários animais silvestres e domésticos, tendo o homem como hospedeiro secundário ${ }^{411}$. A transmissão habitual se dá através da picada de insetos das várias espécies de flebotomíneos, dependendo da localização geográfica $^{10}$. Estima-se que em todo o mundo ocorram dois milhões de novos casos de LTA a cada ano e que $10 \%$ da população mundial esteja sob risco de adoecer ${ }^{42}$.

No Brasil, diminuição gradual da ocorrência da LTA foi registrada a partir dos anos 50 do século XX. No entanto, esse quadro foi interrompido por um crescimento da incidência nos últimos 20 anos, provavelmente relacionado

\footnotetext{
1. Curso de Pós-graduação da Faculdade de Ciências Médicas da Universidade Federal de Mato Grosso, Cuiabá, MT. 2. Secretaria Municipal de Saúde de Chapada dos Guimarães, Chapada dos Guimarães, MT. 3. Hospital Universitário Júlio Muller, Cuiabá, MT.

Endereço para correspondência: Prof. Alex Miranda Rodrigues. Caixa Postal 81, 78195-000 Chapada dos Guimarães, MT.

e-mail: gsveredas@brturbo.com

Recebido para publicação em 20/5/2005

Aceito em 27/1/2006
} 
ao processo de colonização de novas fronteira agrícolas, onde tem destaque as regiões Centro-0este e, mais recentemente, a região Norte ${ }^{4101129}$.

Sua forma cutânea, a mais comum, vem causando significativa morbidade em nosso país, especialmente em áreas rurais, onde o acesso a cuidados de saúde é mais precário, atingindo predominantemente indivíduos do sexo masculino em idade produtiva. Clinicamente, a leishmaniose cutânea (LC) define-se pela presença de lesões que se iniciam no ponto de inoculação das formas infectantes do parasita, através da picada do vetor. A lesão primária é geralmente única, embora eventualmente múltiplas picadas do flebotomíneo ou a disseminação do parasito possam gerar um número elevado de lesões. Surge após um período de incubação variável de 10 dias a três meses, como uma pápula eritematosa que progride lentamente para nódulo. Acompanha-se de adenopatia regional, com ou sem linfangite, em $12 \%$ a $30 \%$ dos casos. Com a evolução, ocorre notável polimorfismo das lesões, sendo possível encontrar formas impetigóide, liquenóide, tuberculóide ou lupóide, nodular, vegetante e ectimatóide. São freqüentes as ulcerações com bordas elevadas, enduradas e fundo com tecido de granulação ${ }^{2461113}$.

Uma grande dificuldade para o tratamento da LTA é gerada pela sua ocorrência em áreas rurais longínquas, com difícil acesso ao serviço de saúde, e a necessidade exclusiva de terapêutica parenteral ${ }^{4617}$. Os antimoniais pentavalentes representam a principal opção terapêutica, sendo utilizado em nosso meio o antimoniato de meglumina como droga de primeira linha ${ }^{491117}$. De acordo com as orientações do Ministério da Saúde, a dose de meglumina a ser empregada para o tratamento da LC é de $10-20 \mathrm{mg} / \mathrm{kg} /$ peso por dia, durante 20 dias, com possibilidade de repetição de um segundo ciclo com duração de 30 dias, em caso de não resolução das lesões, após 90 dias do término do primeiro ciclo ${ }^{91011} 17$.

0 índice de sucesso com o esquema descrito, isto é, cicatrização das lesões dos pacientes tratados após um primeiro ciclo de antimoniato de meglumina, é muito variável na literatura, variando de $26 \%$ a $100 \%$, segundo diferentes autores e em estudos conduzidos em diferentes locais e serviços 317212730 . Essa baixa eficácia representa mais um agravante na operacionalização do tratamento, especialmente para pacientes residentes em áreas afastadas dos centros de saúde ${ }^{4}$. 0 uso de medicamentos alternativos como a anfotericina $B$ e a pentamidina é indicado nos casos de resistência ou impossibilidade de uso do antimoniato de meglumina. Porém, com a limitação de apresentar efeitos colaterais freqüentes e graves, além de alto custo de tratamento e necessidade de serviços de maior complexidade para sua administraçã $0^{410}$.

Embora alguns pesquisadores já tenham identificado baixa eficácia dos antimoniais pentavalentes contra alguns isolados de Leishmania e principalmente para pacientes que apresentam lesões cutâneas múltiplas ou negatividade à reação de Montenegro, outros aspectos relacionados ao paciente e ao uso do esquema de antimoniato de meglumina ainda não foram completamente examinados ${ }^{2024}$. Além disto, apesar da alta endemicidade da LC no Estado de Mato Grosso, nenhuma informação é hoje disponível sobre o perfil de resposta terapêutica dos pacientes desta região, ao tratamento convencional com 0 antimonial pentavalente.

Este trabalho apresenta os resultados de estudo delineado para identificar fatores associados ao insucesso do tratamento da LC, após o primeiro ciclo do esquema de antimoniato de meglumina. A identificação desses fatores pode orientar a formulação de esquemas mais efetivos e seguros para o tratamento da doença, bem como permitir a identificação de grupos de pacientes com padrão específico de resposta ao tratamento de primeira linha, para os quais a opção inicial por esquemas hoje considerados alternativos seja mais adequada.

\section{PACIENTES E MÉTODOS}

Foi constituída uma coorte histórica do período de agosto de 1994 a agosto de 2004, de pacientes com diagnóstico de LC atendidos no Ambulatório de Leishmanioses do Hospital Universitário Julio Muller da Universidade Federal de Mato Grosso (UFMT), localizado em Cuiabá-MT, e que concluíram tratamento com uso de antimoniato de meglumina. Este serviço atua como referência estadual para diagnóstico e tratamento das leishmanioses, recebendo casos de todo o Estado de Mato Grosso e, eventualmente de outros estados das regiões Norte e Centro-Oeste. Atende pacientes procedentes de áreas rurais e, em geral, portadores de quadros mais complexos ou complicados da doença. Dados não publicados dão conta que a espécie de parasita predominante em nosso serviço é a Leishmania (Viannia) brasiliensis, responsável por 91,7\% dos casos (NM Melo: Comunicação pessoal, 2004). 0 presente estudo foi aprovado pelo comitê de ética do Hospital Universitário Julio Muller, sob o número 099-CEP-HUJM/2003.

Pacientes com diagnóstico de LC tratada com antimoniato de meglumina e que concluíram o esquema preconizado pelo Ministério da Saúde foram considerados elegíveis pra o estudo. A confirmação diagnóstica foi feita por exames parasitológicos e/ou por critério clínico-epidemiológico, ou seja, procedência ou residência em área de transmissão da LTA, mais apresentação clínica compatível e positividade da intradermorreação de Montenegro (IRM). Foram excluídos deste estudo pacientes que haviam recebido tratamento completo para LTA com antimoniato de meglumina em outros serviços.

Dos pacientes incluídos foram obtidas informações disponíveis nos prontuários médicos, sobre os aspectos sócio-demográficos, clínicos, laboratoriais, da regularidade e do desfecho do tratamento. As seguintes variáveis foram consideradas como potenciais fatores associados à resposta ao tratamento da LC: gênero, idade, raça, tempo de evolução de sintomas, modalidade de exposição, história de tratamento anterior para leishmaniose, número de lesões cutâneas, regularidade do tratamento, resultado da IRM, peso corporal, dose de antimoniato de meglumina por quilograma de peso e efeitos colaterais relatados pelos pacientes e/ou observados durante o acompanhamento do tratamento. Todos os pacientes receberam tratamento ambulatorial supervisionado 
com antimoniato de meglumina, que também foi indicado em caso de não resposta ao primeiro esquema, para a maioria dos pacientes que recrudesceu.

Definiu-se como sucesso terapêutico, ou resposta terapêutica satisfatória, a cicatrização completa da lesão cutânea provocada pela LC, em no máximo três meses de observação após o tratamento com o primeiro esquema de antimoniato de meglumina, nas doses preconizadas pelo Ministério da Saúde. O insucesso foi definido como a não cicatrização da lesão cutânea ou a sua recrudescência durante os três meses de seguimento após o término do primeiro esquema terapêutico, independente da regularidade do tratamento.

A regularidade do tratamento foi categorizada como tratamento regular quando o paciente recebeu o esquema proposto de 20 dias de tratamento, com sua conclusão em no máximo 30 dias e sem falta por período superior a dois dias consecutivos $^{10}$. Pacientes que completaram $50 \%$ a $90 \%$ da dose total de meglumina prescrita, e/ou tiveram faltas consecutivas superiores a dois dias, e/ou concluíram o esquema em mais de 30 dias, foram categorizados com tendo tratamento irregular.

Foram descritos os dados referentes aos efeitos tóxicos observados e relatados pelos pacientes incluídos nesta coorte. Os resultados de amilase, aspartato aminotransferase (ALT), alanina aminotransferase (AST), fosfatase alcalina, uréia e creatinina, que são solicitados rotineiramente no serviço, foram resgatados dos prontuários. Os eletrocardiogramas foram lidos por um pesquisador que não estava ciente do momento em que o traçado fora obtido. Foram medidos os intervalos entre as ondas $\mathrm{R}(\mathrm{RR})$ e entre a onda $\mathrm{Q}$ e a onda T (QT) para determinação do intervalo corrigido entre a onda Q e a anda T (QTc), de acordo com fórmula de Bazzet.

Os dados foram tabulados e analisados com auxílio dos softwares Epi-Info versão 6,04, 2001 e Stata versão 5,0. Variáveis contínuas foram categorizadas de acordo com critério estatístico (peso), farmacológico (dose diária de antimônio) ou clínico (idade, número de lesões e tempo de evolução). Os dados laboratoriais foram analisados pelo teste de $t$ pareado, comparando-se os resultados dos exames realizados antes e durante o tratamento com antimoniato de meglumina. As incidências de sucesso ou falha terapêutica foram calculadas para cada uma das variáveis estudadas e o risco relativo de insucesso terapêutico, bem como o seu intervalo de confiança (IC 95\%), foram determinados. Os fatores independentemente associados ao insucesso terapêutico foram avaliados usando um modelo de regressão logística, com mecanismo automático (stepwise-forward) de inclusão das variáveis ${ }^{16}$. Como nesse modelo de análise a variável dose de antimonial por quilograma de peso foi excluida automaticamente, um novo modelo de análise multivariada foi construído, com inclusão manual de todas as variáveis que permaneceram estatisticamente associadas ao insucesso terapêutico, ao nível de significância de 90\% $(\mathrm{p}<0,10)$, mais sexo e idade, dado à influência destas variáveis nos desfechos dos eventos biológicos. Na análise multivariada, a força de associação foi determinada pelo cálculo do odds ratio e IC95\%, utilizando o método de Wolf ${ }^{15}$.

\section{RESULTADOS}

Foram incluídos na coorte histórica 151 pacientes, sendo $131(86,8 \%)$ do sexo masculino, com idade variando de 1 a 69 anos (média $\pm \mathrm{DP}=34,0 \pm 13,9$ anos). Diagnóstico parasitológico foi confirmado em 113 (74,8\%) pacientes e o número de lesões cutâneas variou de 1 a 18 (mediana=1), com tempo de evolução de 0,5 a 36 (mediana $=2,5)$ meses. A dose diária média $( \pm \mathrm{DP})$ de antimoniato de meglumina administrada foi de 13,3 $( \pm 2,7) \mathrm{mg} / \mathrm{kg} / \mathrm{dia}$.

Após análise da coorte pós-tratamento, os pacientes foram classificados em dois grupos: de insucesso terapêutico $(n=71)$, representando incidência de $47 \%$ (IC95: $39,2 \%-55 \%)$ de falha, e de sucesso terapêutico $(n=80)$, representando incidência de 53\% (IC95:50\%-60,8\%) de cura, após um primeiro ciclo de antimoniato de meglumina. A Tabela 1 apresenta as incidências de insucesso do tratamento de acordo com variáveis sócio-demográficas e clínicas. Não houve diferença das distribuições de idade, raça, positividade à IRM, efeitos adversos e tempo de evolução da doença, entre os grupos estudados.

Os principais efeitos adversos observados e relatados estão apresentados na Tabela 2. Noventa (59,6\%) pacientes apresentaram pelo menos um sinal ou sintoma atribuído a efeito colateral. Os sintomas ou sinais mais relatados durante o tratamento foram artralgia $(25,8 \%)$, mialgia $(22,5 \%)$, cefaléia $(13,3 \%)$, fraqueza $(7,9 \%)$, febre $(6,6 \%)$, e epigastralgia $(6,6 \%)$. Não foram observadas diferenças significativas, entre os grupos, nos resultados das medidas de amilase, enzimas hepáticas, uréia, creatinina e fosfatase alcalina, avaliadas antes e durante o tratamento da LC. 0 traçado eletrocardiográfico obtido durante o tratamento foi realizado após tempo médio $( \pm \mathrm{DP})$ de 10,8 $( \pm 4,1)$ dias do início da terapêutica. Nesse período, observou-se incremento significativo do QTc e em $18(22,5 \%)$ pacientes esse intervalo ultrapassou o limite de 0,440 segundos. No entanto, o incremento médio \pm DP do QTc observado durante o tratamento, em relação ao traçado feito antes do tratamento, foi $0,027 \pm 0,033$ segundos, e não influenciou o desfecho terapêutico $(\mathrm{p}=0,89)$.

0 insucesso do tratamento esteve associado à dose de antimoniato de meglumina inferior a $10 \mathrm{mg} / \mathrm{kg} / \mathrm{dia}(\mathrm{RR}=1,8$; IC95:1,1-3,0), ao tratamento prévio para LTA $(R R=1,7$; IC95:1,3-2,4), ao numero de lesões superior a $3(\mathrm{RR}=1,9$; IC95:1,4-2,5), a tratamento irregular ( $R R=1,9$; IC95:1,3$2,6)$ e ao peso maior que 68kg ( $R R=1,7$; IC95:1,1-2,5). Após o ajuste em análise multivariada, apenas o peso maior que $68 \mathrm{Kg}(\mathrm{OR}=4,3$; IC95\%=1,5-11,9), o numero de lesões maior que $3(\mathrm{OR}=4,6$; IC $95 \%=1,2-17,4)$, o tratamento irregular $(\mathrm{OR}=12,5 ; \mathrm{IC} 95 \%=2,1-75,4)$ e a história de tratamento anterior para LTA $(\mathrm{OR}=4,5$; IC95\% $=1,1-17,5)$ permaneceram associados ao insucesso do tratamento (Tabela 3). 
Tabela 1 - Características clínicas e sócio-demográficas associadas ao insucesso do tratamento da forma cutânea da leishmaniose tegumentar americana com antimoniato de meglumina.

\begin{tabular}{|c|c|c|c|c|c|}
\hline Características & Número de pacientes & Insucesso terapêutico & Sucesso terapêutico & Risco relativo (IC95\%) & $\mathrm{p}$ \\
\hline \multicolumn{6}{|l|}{ Sexo } \\
\hline masculino & 131 & 66 & 65 & $2,01(0,93-4,39)$ & 0,03 \\
\hline feminino & 20 & 5 & 15 & 1,0 & \\
\hline \multicolumn{6}{|l|}{ Idade (anos) } \\
\hline$\geq 60$ & 7 & 4 & 3 & $1,23(0,63-2,39)$ & 0,58 \\
\hline$<60$ & 144 & 67 & 77 & 1,0 & \\
\hline \multicolumn{6}{|c|}{ Dose de antimoniato de meglumina (mg/kg/dia) } \\
\hline$<10$ & 12 & 9 & 3 & $1,81(1,11-2,95)$ & 0,04 \\
\hline $10-15$ & 80 & 37 & 43 & $1,12(0,72-1,72)$ & 0,62 \\
\hline$>15$ & 41 & 17 & 24 & 1,0 & \\
\hline Média \pm DP & 133 & $12.9 \pm 3.2$ & $13.8 \pm 2.1$ & - & 0.09 \\
\hline \multicolumn{6}{|c|}{ Número de lesões } \\
\hline$\geq 3$ & 28 & 21 & 7 & $1,85(1,36-2,49)$ & 0,001 \\
\hline$<3$ & 123 & 50 & 73 & 1,0 & \\
\hline \multicolumn{6}{|c|}{ Tempo de evolução da doença (meses) } \\
\hline$<3$ & 65 & 31 & 34 & 1,0 & \\
\hline 3 a 5 & 52 & 19 & 33 & $0,77(0,49-1,19)$ & 0,23 \\
\hline 6 a 11 & 18 & 11 & 7 & $1,28(0,82-2,01)$ & 0,31 \\
\hline$\geq 12$ & 4 & 3 & 1 & $1,57(0,85-2,92)$ & 0,29 \\
\hline \multicolumn{6}{|c|}{ História de tratamento anterior para LTA } \\
\hline $\operatorname{sim}$ & 24 & 18 & 6 & $1,72(1,26-2,36)$ & 0,005 \\
\hline não & 108 & 47 & 61 & 1,0 & \\
\hline \multicolumn{6}{|l|}{ Raça } \\
\hline branca & 47 & 28 & 19 & $1,29(0,89-1,87)$ & 0,18 \\
\hline não branca & 54 & 25 & 29 & 1,0 & \\
\hline \multicolumn{6}{|l|}{ IRM } \\
\hline positiva & 102 & 42 & 60 & $0,82(0,42-1,60)$ & 0,59 \\
\hline negativa & 10 & 5 & 5 & 1,0 & \\
\hline \multicolumn{6}{|c|}{ Tratamento irregular } \\
\hline $\operatorname{sim}$ & 12 & 10 & 2 & $1,85(1,33-2,56)$ & 0,01 \\
\hline não & 113 & 51 & 62 & 1,0 & \\
\hline \multicolumn{6}{|c|}{ Efeitos adversos incidentes } \\
\hline $\operatorname{sim}$ & 90 & 37 & 53 & $0,55(0,27-1,13)$ & 0,08 \\
\hline não & 61 & 34 & 27 & 1,0 & \\
\hline \multicolumn{6}{|l|}{ Peso (kg) } \\
\hline$>68$ & 69 & 42 & 27 & $1,67(1,12-2,49)$ & 0,007 \\
\hline$\leq 68$ & 55 & 20 & 35 & 1,0 & \\
\hline
\end{tabular}

Tabela 2 - Principais efeitos adversos observados e relatados em 151 pacientes tratado para leishmaniose cutânea com antimoniato de meglumina.

\begin{tabular}{lrr}
\hline & \multicolumn{3}{c}{ Pacientes } \\
\cline { 2 - 3 } Efeito colateral & $\mathrm{n}^{0}$ & $\%$ \\
\hline Artralgia & 39 & 25,8 \\
Mialgia & 34 & 22,5 \\
Cefaléia & 20 & 13,3 \\
Fraqueza & 12 & 7,9 \\
Febre & 10 & 6,6 \\
Epigastralgia & 10 & 6,6 \\
Plenitude pós prandial & 8 & 5,3 \\
Inapetência & 7 & 4,6 \\
Náuseas & 7 & 4,6 \\
Tonteira & 6 & 4,0 \\
Prurido & 5 & 3,3 \\
Qualquer efeito colateral & 90 & 59,6 \\
Nenhum efeito colateral & 61 & 40,4 \\
\hline
\end{tabular}

Tabela 3 - Resultado da análise multivariada dos fatores associados ao insucesso do tratamento da forma cutânea da leishmaniose tegumentar americana com antimoniato de meglumina.

\begin{tabular}{lrr}
\hline Fator & OR (IC 95\%) & $\mathrm{p}$ \\
\hline Peso superior a 68 kg & $4,3(1,5-11,9)$ & 0,005 \\
Tratamento irregular & $12,5(2,1-75,4)$ & 0,006 \\
História de tratamento anterior para LTA & $4,5(1,1-17,5)$ & 0,031 \\
Número de lesões > 3 & $4,6(1,2-17,4)$ & 0,023 \\
\hline Foram incluídas no modelo de análise multivariada as seguintes variáveis: sexo, idade, \\
$\begin{array}{l}\text { dose por kg de peso, número de lesões, tratamento anterior para LTA, efeitos adversos } \\
\text { incidentes, tratamento regular para LTA e peso. }\end{array}$
\end{tabular}

\section{DISCUSSÃo}

No presente estudo, observou-se uma incidência de insucesso de $47 \%$ (IC95:39,2\%-55,0\%), consistente com os dados observados na literatura que, embora muito variáveis, dão conta que a proporção de pacientes que não responde a este grupo de drogas varia de zero a $74 \%$, e que fatores 
relacionados ao hospedeiro, ao parasita e à utilização da medicação são intervenientes nesse processo $0^{3820242526}$.

$\mathrm{Na}$ presente coorte, fatores sócio-demográficos, clínicos, e relacionados à utilização do antimonial pentavalente contribuíram para o desfecho terapêutico da LC. A história de tratamento anterior para LTA, tratamento irregular, um maior número de lesões cutâneas e o peso do paciente superior a $68 \mathrm{~kg}$ são características que conferiram maior risco de insucesso ao tratamento. Não houve associação entre o resultado da IRM, ocorrência de efeito colateral ou dose de antimoniato de meglumina com a ocorrência de insucesso ao tratamento.

Embora tenham sido excluídos pacientes que referiram ter recebido tratamento completo com antimoniato de meglumina previamente à sua inclusão no estudo, a associação do insucesso do tratamento com a história de tratamento anterior para LTA pode ser consequiência de uso de dose insuficiente da medicação, promovendo a seleção de parasita resistente aos antimoniais pentavalentes. De fato, o surgimento de resistência aos compostos antimoniais tem sido demonstrado in vitro e em animais naturalmente infectados, durante exposição curta a essa classe de drogas, ou a esquemas terapêuticos que utilizaram dose total de antimônio baixa ${ }^{12} 14$.

Já foi demonstrado que determinadas espécies de Leishmania, como a L. (V.) guyanensis, causam formas cutâneas de LTA com maior número de lesões e são significativamente mais resistentes ao tratamento com antimoniais ${ }^{2526}$. Esse achado poderia justificar a associação entre a maior ocorrência de insucesso do tratamento da LTA com antimoniato de meglumina e o número de lesões cutâneas. Infelizmente, não foi possível a caracterização das espécies de Leishmania envolvidas na patologia dos pacientes incluídos nesta coorte. Por outro lado, fatores relacionados à resposta do hospedeiro não puderam ser completamente descartados neste estudo.

0 tratamento irregular demonstrou uma previsível associação com o insucesso terapêutico. É sabido que eficácia dos compostos antimoniais é obtida com doses altas dos medicamentos e por período prolongado de administração ${ }^{317}$. Se administrados em períodos mais curtos, os esquemas de antimoniais necessitam ser repetidos em novos ciclos, como eram preconizados anteriormente, nas normas brasileiras de tratamento das leishmanioses ${ }^{3}$. Outra explicação seria a ocorrência de efeitos adversos, levando a interrupções temporárias do tratamento durante o período do primeiro ciclo. No entanto, a incidência de efeitos colaterais não se relacionou com a irregularidade ou com o insucesso do tratamento nesta coorte.

Embora as normas brasileiras e internacionais recomendem doses de antimoniais superiores a $10 \mathrm{mg} / \mathrm{kg} /$ dia, usualmente mais próximas a $20 \mathrm{mg} / \mathrm{kg} / \mathrm{dia}$, especialmente nas formas clínicas mais graves da doença ${ }^{5}{ }^{10}$, na coorte estudada não foi possível determinar associação entre a dose de antimonial e o insucesso do tratamento da LTA. Por outro lado, recentes estudos não relacionaram doses menores que $10 \mathrm{mg} / \mathrm{kg} /$ dia ao insucesso do tratamento da LTA em análise retrospectiva ${ }^{128}$ e demonstraram bons resultados com doses de antimoniais inferiores a $10 \mathrm{mg} / \mathrm{kg} / \mathrm{dia}$, mesmo com longos períodos de seguimento, em estudos prospectivos observacionais ${ }^{18}{ }^{19}$. É possível que o número de pacientes arrolados no presente estudo não tenha sido suficientemente grande para mostrar a associação entre a dose de antimoniato de meglumina e o desfecho terapêutico. Corrobora essa hipótese a maior incidência de insucesso no grupo de pacientes com peso maior que $68 \mathrm{~kg}$. Isto pode ter sido devido à tendência de limitar-se a dose máxima de antimoniato de meglumina a 3 ampolas por dia (1.215mg de antimônio), segundo as normas brasileiras de controle da $\mathrm{LTA}^{910}$. Não sendo, portanto uma real influência do peso. Além disto, como esta pesquisa baseou-se em uma coorte histórica e não em um estudo prospectivo com dose fixa por peso, é possível que pacientes com obesidade e suas comorbidades cardiovasculares tenham influenciado na homogeneidade das doses prescritas. É razoável pensar que os médicos que atenderam tais pacientes tendiam a usar limites inferiores de dose recomendada por peso, nos cálculos dos pacientes mais obesos, seja pelos riscos associados ao aumento da dose diária de antimonial pentavalente acima de três ampolas por dia, ou pelo maior risco de toxicidade para pacientes portadores de outras patologias. De fato, os pacientes que representaram os $25 \%$ mais pesados da amostra, receberam, em média, menor dose de animoniato de meglumina por quilograma de peso do que os demais, embora esta diferença não tenha sido estatisticamente significante $(p=0,06)$. Não se pode também descartar a influência de fatores farmacológicos ainda não descritos, que justifiquem a associação entre peso e insucesso do tratamento da LTA com antimoniato de meglumina, uma vez que o desenvolvimento e a pesquisa de drogas para o tratamento deste grupo de patologias são freqüentemente negligenciados $^{1417}$. 0 impacto de diferentes dosagens de antimonial sobre a eficácia, embora não tenha sido demonstrado nesta coorte, pode refletir a ocorrência de espécies de Leishmania com diferentes perfis de sensibilidade a esse grupo de drogas, o que já vem sendo observado há muitos anos ${ }^{26}$, levando inclusive alguns pesquisadores a sugerir a utilização de esquemas com dosagens individualizadas, de acordo com a síndrome clínica e espécie envolvidas ${ }^{17}$. Contudo, o resultado observado neste coorte pode sugerir que as espécies e/ou isolados de Leishmania que circulam em nossa região são menos susceptíveis a dosagens baixas de antimoniato de meglumina, embora estudos observacionais com um maior número de pacientes e ensaios clínicos prospectivos sejam mais adequados para elucidar tais aspectos da terapêutica.

Embora, outros pesquisadores tenham demonstrado o valor da negatividade da intradermorreação de Montenegro como preditor de insucesso terapêutico da LTA, porém com desfecho direcionado para a recorrência de lesão num período de acompanhamento de dois anos ${ }^{20}$, o mesmo achado não foi observado em nosso grupo de pacientes. As explicações para esse achado seriam o desfecho de nosso estudo ser direcionado para a cicatrização ou não das lesões ao final de no máximo 3 meses de observação, bem como um significativo número de pacientes arrolados em nosso estudo apresentando doença precoce, período em que a maioria dos pacientes ainda não apresenta a positivação da intradermorreação de Montenegro ${ }^{7}$. 
Um possível viés de seleção do presente estudo é ter incluído pacientes exclusivamente de um serviço de referência. Neste sentido, a proporção de $47 \%$ de insucessos terapêuticos encontrada nesta coorte, embora não diferente da observada por outros pesquisadores, deve ser considerada com reservas como reflexo da proporção real de insucesso terapêutico para LTA em nossa região. Em serviços de referência há, em geral, uma demanda de casos mais graves ou complicados, prejudicando a extrapolação de suas características para pacientes atendidos em serviços de menor complexidade do sistema de saúde, como já anteriormente demonstrado ${ }^{23}$.

Os resultados do presente estudo permitem identificar pacientes em que o tratamento com antimoniais pentavalentes pode ser menos eficaz, a partir de variáveis clínicas como peso, número de lesões cutâneas e antecedente de uso de medicação antimonial, bem como prever um pior resultado para aqueles que concluíram o esquema terapêutico de modo irregular. A incorporação desse conhecimento nas normas de tratamento da LTA, ou em eventuais algorítimos clínicos, pode orientar com mais clareza a formulação de esquemas de tratamentos mais efetivos para a doença, baseados apenas em características individuais do paciente.

\section{AGRADECIMENTOS}

Ao Professor Dr. Francisco José Dutra Souto, pela leitura e sugestões durante elaboração do manuscrito. A estudante de medicina Andréia Ferreira Néry e técnico Bruno Santos de Souza, pelo auxílio no levantamento de dados dos prontuários.

\section{REFERENCIAS BIBLIOGRÁFICAS}

1. Andrade MS, Brito MEF, Silva ST, Lima BS, Almeida EL, Albuquerque EL, Marinho Junior JF, Ishikawa E, Cupolillo E, Brandao Filho JF. Leishmaniose Tegumentar Americana causada por Leishmania (Viannia) braziliensis em uma área de treinamento militar na Zona da Mata de Pernambuco. Revista da Sociedade Brasileira de Medicina Tropical 38:229-233, 2005

2. Andrade-Narvaez FJ, Vargas-Gonzalçes A, Canto-Lara SB, Damián-Centeno AB. Clinical picture of cutaneous leishmaniases due to Leishmania (Leishmania) mexicana in the Yucatan peninsula, Mexico. Memórias do. Insituto Oswaldo Cruz 96:163-167, 2001.

3. Azeredo-Coutinho RBG, Mendonça SCF. O esquema de tratamento antimonial intermitente é superior ao contínuo na terapia da leishmaniose cutânea no município do Rio de Janeiro, Brasil. Revista da Sociedade Brasileira de Medicina Tropical 35: 477-481, 2002

4. Basano SA, Camargo LMA. Leishmaniose tegumentar americana: história, epidemiologia and perspectivas de controle. Revista Brasileira de Epidemiologia 7:328-337, 2004.

5. Blum J, Desjeux P, Schwartz E, Beck B, Hatz C. Tratament of cutaneous leishmaniasis among travellers. Journal of Antimicrobial Chemotherapy 53: 158-166, 2004.

6. Castro EA, Soccol VT, Membrive N, Luz N. Epidemiological and clinical study of 332 cases of cutaneous leishmaniasis in the north of Parana State from 1993 to 1998. Revista da Sociedade Brasileira de Medicina Tropical 35: 445-452, 2002.

7. Cuba-Cuba CA, Marsden PD, Barretto AC, Jones TC, Richards F. The use of different concentrations of leishmanial antigen in skin testing to evaluate delaved-hypersensitivity in American cutaneous leishmaniasis. Revista da Sociedade Brasileira de Medicina Tropical 18:231-236, 1985.

8. Deps PD, Viana MC, Falqueto A, Dietze R. Evaluation of the efficacy and toxicity of N-methyl-glucamine vs $\mathrm{BP} 88^{\circledR}$ Sodium Stibogluconate in the treatment of localized cutaneous leishmaniasis. Revista da Sociedade Brasileira de Medicina Tropical 33:535-543, 2000.

9. Fundação Nacional de Saúde. Doenças infecciosas e parasitarias: aspectos clínicos, de vigilância epidemiológica e de controle. Ministério da Saúde, Brasília, 1998.

10. Fundação Nacional de Saúde. Manual de controle da leishmaniose tegumentar Americana. Ministério da Saúde, Brasília, 2000.

11. Gontijo B, Carvalho MLR. American cutaneous leishmaniasis. Revista da Sociedade Brasileira de Medicina Tropical 36:71-80, 2003.

12. Grogl M, Thomason TN, Franke ED. Drug resistance in leishmanisis: its implication in systemic chemotherapy of cutaneous and mucocutaneous disease. The American Journal of Tropical Medicine and Hygiene 47:117$126,1992$.

13. Guerra JAO, Talhari S, Paes MG, Garrido M, Talhari JM. Aspectos clínicos e diagnósticos da leishmaniose tegumentar americana em militares simultaneamente expostos à infecção na Amazônia. Revista da Sociedade Brasileira de Medicina Tropical 36: 587-590, 2003.

14. Herwaldt BL. Leishmaniasis. Lancet 354:1191-1199, 1999.

15. Hosmer DW, Lemenshow S. Applied logistic regression. Johns Wiley and Sons, New York, 1989.

16. Katz MH. Multivariable Analysis: A primer for reader of medical research. Annals of Internal Medicine 138:644-650, 2003.

17. Marsden PD. Pentavalent antimonials: old for new diseases. Revista da Sociedade Brasileira de Medicina Tropical 18:187-198, 1985.

18. Oliveira-Neto MP, Mattos M, Pirmez C. Mucosal leishmaniasis ("espundia”) responsive to low dose of $\mathrm{N}$-methyl glucamine (Glucantime ${ }^{\circledR}$ ) in Rio de Janeiro, Brazil. Revista do Instituto de Medicina Tropical de São Paulo 42:321-325, 2000.

19. Oliveira-Neto MP, Schubach A, Mattos M, Gonçalves-Costa SC, Pirmez C. A low-dose antimony treatment in 159 patients with american cujtaneous leishmaniasis: extensive follow up studies (up to 10 years). The American Journal of Tropical Medicine and Hygiene 57:651-655, 1997.

20. Passos VAM, Barreto SM, Romanha AJ, Krettli AU, Volpini AC, Costa MFFL. American cutaneous leishmaniasis: use of a skin test as a predictor of relapse after treatment. Bulletin of the World Health Organization 78:968$974,2000$.

21. Passos VAM, Barreto SM, Romanha AJ, Krettli AU, Volpini AC, Gontijo CMF, Falcão AL, Costa MFFL. Leishmaniose tegumentar na região metropolitana de Belo Horizonte: aspectos clínicos, laboratoriais, terapêuticos e evolutivos (1989-1995). Revista da Sociedade Brasileira de Medicina Tropical 34:5-12, 2001.

22. Roberts LJ, Handman E, Foote SJ. Leishmaniasis. British Medical Journal 321: 801-804, 2000

23. Rodrigues AM, Fontes CJF. Insucesso e toxicidade no tratamento da leishmaniose tegumentar americana: efeitos de viés de seleção. In: Resumos do XL Congresso da Sociedade Brasileira de Medicina Tropical, Aracaju, p. 15,2004

24. Romero GAS. Predicting relapse after treatment for american cutaneous leishmaniasis. Bulletin of the World Health Organization 79: 1169, 2001.

25. Romero GAS, Guerra MVF, Paes MG, Macedo VO. Comparison of cutaneous leishmaniasis due to Leishmania (Viannia) braziliensis and $L$. (V.) guyanensis in Brazil: clinical findings and diagnostic approach. Clinical of Infectious Diseases 32:1304-1312, 2001.

26. Romero GAS, Guerra MVF, Paes MG, Macedo VO. Comparison of cutaneous leishmaniasis due to Leishmania (Viannia) braziliensis and $L$. (V.) guyanensis in Brazil: Therapeutic response to meglumine antimoniate. American Journal of Tropical Medicine and Hygiene 65:456-465, 2001.

27. Saldanha ACR, Romero GAS, Merchan-Hamann E, Magalhães AV, Macedo VO. Comparative study between sodium stibogluconate BP88® and meglumine antimoniate for cutaneous leishmaniasis treatment: I. Efficacy 
and safety. Revista da Sociedade Brasileira de Medicina Tropical 32:383$387,1999$.

28. Schubach, AO, Marzochi KBF, Moreira JS, Schubach TMP, Araujo ML, Vale ACF, Passos SRL, Marzochi MCA. Estudo retrospectivo de 151 pacientes com leishmaniose cutânea tratados com antimoniato de meglumina. Revista da Sociedade Brasileira de Medicina Tropical 38:213-217, 2005.
29. Silva NS, Viana AB, Cordeiro JA, Cavasini CE. American cutaneous leishmaniasis in the State of Acre, Brazil. Revista de Saúde Pública 33:554559, 1999.

30. Soto J, Toledo J, Vega J, Berman J. Efficacy of pentavalent antimony for treatment of colombian cutaneous leishmaniasis. The American Journal of Tropical Medicine and Hygiene 72:421-422, 2005. 